\title{
Hyers-Ulam Stability of Generalized Tribonacci Functional Equation
}

\author{
S. Arolkar ${ }^{1, *}$, Y.S. Valaulikar ${ }^{2}$ \\ ${ }^{1}$ Department of Mathematics, D.M.'s College and Research Centre, Assagao, Goa 403 507- India \\ ${ }^{2}$ Department of Mathematics, Goa University, Taleigao Plateau, Goa 403206 - India \\ *Corresponding author: Suchita.golatkar@yahoo.com \\ Received November 14, 2016; Revised March 06, 2017; Accepted March 23, 2017
}

\begin{abstract}
In this paper we study Hyers-Ulam stability of the generalized Tribonacci functional equation, $f(x)=a f(x-1)+b f(x-2)+c f(x-3)$, where $a, b$ and $c$ are non- zero constants. The functional equation is solved and its stability is established in the class of functions $f: \mathbb{R} \rightarrow X$, where $X$ is a Banach space.
\end{abstract}

Keywords: Fibonacci functional equation, Hyers-Ulam stability, Tribonacci functional equation

Cite This Article: S. Arolkar, and Y.S. Valaulikar, "Hyers-Ulam Stability of Generalized Tribonacci Functional Equation." Turkish Journal of Analysis and Number Theory, vol. 5, no. 3 (2017): 80-85. doi: 10.12691/tjant-5-3-1.

\section{Introduction}

Stability problems of functional equations have been extensively studied. (see $[1,5,6]$ and references therein). The importance of the topic lies in the fact that stability of functional equation is associated with notions of Controlled Chaos [11] and Shadowing [14]. In [7] and [8] Jung discusses the stability problem for Fibonacci functional equation and its extension in Banach Space respectively whereas in [3] the problem is discussed in Modular Functional space. In [10] $k$-Fibonacci functional equation is discussed. In [2] and [4] solution and stability of Tribonacci functional equation in non-Archimedean Banach spaces and 2-normed spaces have been discussed respectively. Stability of Tribonacci and k-Tribonacci functional equations in Modular spaces is discussed in [13]. In [9], authors investigate the solution of generalized linear Tribonacci functional equation in terms of Fibonacci numbers. In this paper we show that the generalized linear Tribonacci functional equation is associated with generalized Tribonacci sequence and also obtain its stability in the class of functions $f: \mathbb{R} \rightarrow X$, where $X$ is a real (or complex) Banach space. Like Fibonacci numbers, Tribonacci numbers also play an important role in problems of combinatorics [12] and also in evaluation of determinants of circulant matrices [15]. We now define the generalized Tribonacci sequence.

Definition 1. The generalized Tribonacci sequence is defined by

$$
\begin{gathered}
T_{n+2}=a T_{n+1}+b T_{n}+c T_{n-1}, \\
T_{0}=0, T_{1}=0 \text { and } T_{2}=1, \text { for all } n \in \mathbb{Z},
\end{gathered}
$$

where $a, b$ and $c$ are non-zero fixed real numbers and $T_{n}$ is the $n^{\text {th }}$ term of (1.1) given by Binet type formula,

$$
T_{n}=\frac{(\alpha-\beta) \gamma^{n}-(\alpha-\gamma) \beta^{n}+(\beta-\gamma) \alpha^{n}}{(\alpha-\beta)(\beta-\gamma)(\alpha-\gamma)} .
$$

where $\alpha, \beta$ and $\gamma$ are distinct roots of the characteristics equation corresponding to (1.1).

Definition 2. Let $X$ be a real (or complex) Banach space. A function $f: \mathbb{R} \rightarrow X$ is called a generalized Tribonacci function if it satisfies generalized Tribonacci functional equation

$$
f(x)=a f(x-1)+b f(x-2)+c f(x-3), \forall x \in \mathbb{R} .(1.3)
$$

\section{Existence of General Solution to the Equation (1.3)}

In this section we solve equation (1.3) and prove that its solution is in terms of (1.2). To prove this result, we require the following lemma.

Lemma 3. If $\alpha, \beta$ and $\gamma$ are distinct roots of the characteristics equation corresponding to (1.1), then the generalized Tribonacci function $f: \mathbb{R} \rightarrow X$ satisfies

$$
\begin{aligned}
& f(x)=T_{n+2} f(x-n) \\
& \quad+\left(b T_{n+1}+c T_{n}\right) f(x-n-1)+c T_{n+1} f(x-n-2),
\end{aligned}
$$

$\forall x \in \mathbb{R}$ and $\forall n \in \mathbb{Z}$.

Proof. Characteristics equation corresponding to (1.1) is

$$
\lambda^{3}-a \lambda^{2}-b \lambda-c=0 .
$$

Since $\alpha, \beta$ and $\gamma$ are distinct roots of (2.2), we get $a=\alpha+\beta+\gamma, b=-(\alpha \gamma+\beta \gamma+\alpha \beta)$ and $c=\alpha \beta \gamma$.

Substituting $a, b$ and $\mathrm{c}$ in (1.3), we have

$$
\begin{aligned}
& f(x)=(\alpha+\beta+\gamma) f(x-1) \\
& -(\alpha \gamma+\beta \gamma+\alpha \beta) f(x-2)+(\alpha \beta \gamma) f(x-3),
\end{aligned}
$$


which implies

$$
\begin{aligned}
& f(x)-(\alpha+\beta) f(x-1)+(\alpha \beta) f(x-2) \\
& =\gamma(f(x-1)-(\alpha+\beta) f(x-2)+(\alpha \beta) f(x-3)) .
\end{aligned}
$$

Replacing $x$ by $x-1$ in (2.3), we get

$$
\begin{aligned}
& f(x-1)-(\alpha+\beta) f(x-2)+(\alpha \beta) f(x-3) \\
& =\gamma(f(x-2)-(\alpha+\beta) f(x-3)+(\alpha \beta) f(x-4))
\end{aligned}
$$

and hence (2.3) yields

$$
\begin{aligned}
& f(x)-(\alpha+\beta) f(x-1)+(\alpha \beta) f(x-2) \\
& =\gamma^{2}(f(x-2)-(\alpha+\beta) f(x-3)+(\alpha \beta) f(x-4)) .
\end{aligned}
$$

By induction on $\mathrm{n}$, we get

$$
\begin{aligned}
& f(x)-(\alpha+\beta) f(x-1)+(\alpha \beta) f(x-2) \\
& =\gamma^{n}(f(x-n)-(\alpha+\beta) f(x-n-1)+(\alpha \beta) f(x-n-2)) .
\end{aligned}
$$

Similarly, we have

$$
\begin{aligned}
& f(x)-(\alpha+\gamma) f(x-1)+(\alpha \gamma) f(x-2) \\
& =\beta^{n}(f(x-n)-(\alpha+\gamma) f(x-n-1)+(\alpha \gamma) f(x-n-2))
\end{aligned}
$$

and

$$
\begin{aligned}
& f(x)-(\beta+\gamma) f(x-1)+(\beta \gamma) f(x-2) \\
& =\alpha^{n}(f(x-n)-(\beta+\gamma) f(x-n-1)+(\beta \gamma) f(x-n-2)),
\end{aligned}
$$

$\forall x \in \mathbb{R}$ and $\forall n \in \mathbb{N} \cup\{0\}$.

Now replacing $x$ by $x+1$ in (2.3), we get

$$
\begin{aligned}
& f(x+1)-(\alpha+\beta) f(x)+(\alpha \beta) f(x-1) \\
& =\gamma(f(x)-(\alpha+\beta) f(x-1)++(\alpha \beta) f(x-2)) .
\end{aligned}
$$

Therefore,

$$
\begin{aligned}
& f(x)-(\alpha+\beta) f(x-1)+(\alpha \beta) f(x-2) \\
& =\gamma^{-1}(f(x+1)-(\alpha+\beta) f(x)+(\alpha \beta) f(x-1))
\end{aligned}
$$

Thus by induction on $\mathrm{n}$, we get

$$
\begin{aligned}
& f(x)-(\alpha+\beta) f(x-1)+(\alpha \beta) f(x-2) \\
& =\gamma^{-n}(f(x+n)-(\alpha+\beta) f(x+n-1)+(\alpha \beta) f(x+n-2)) .
\end{aligned}
$$

Similarly, we have

$$
\begin{aligned}
& f(x)-(\alpha+\gamma) f(x-1)+(\alpha \gamma) f(x-2) \\
& =\beta^{-n}(f(x+n)-(\alpha+\gamma) f(x+n-1)+(\alpha \gamma) f(x+n-2))
\end{aligned}
$$

and

$$
\begin{aligned}
& f(x)-(\beta+\gamma) f(x-1)+(\beta \gamma) f(x-2) \\
& =\alpha^{-n}(f(x+n)-(\beta+\gamma) f(x+n-1)+(\beta \gamma) f(x+n-2)),
\end{aligned}
$$

$\forall x \in \mathbb{R}$ and $\forall n \in \mathbb{N} \bigcup\{0\}$.
Therefore equations (2.4), (2.5) and (2.6) are true $\forall x \in \mathbb{R}$ and $\forall n \in \mathbb{Z}$.

Now multiplying the equations (2.4), (2.5) and (2.6) by $\gamma^{2}(\alpha-\beta),-\beta^{2}(\alpha-\gamma), \quad \alpha^{2}(\beta-\gamma)$ respectively, and adding we get

$f(x)$

$=\left(\frac{\gamma^{n+2}(\alpha-\beta)-\beta^{n+2}(\alpha-\gamma)+\alpha^{n+2}(\beta-\gamma)}{(\alpha-\beta)(\beta-\gamma)(\alpha-\gamma)}\right) f(x-n)$

$+\left(\begin{array}{c}b \frac{\gamma^{n+1}(\alpha-\beta)-\beta^{n+1}(\alpha-\gamma)+\alpha^{n+1}(\beta-\gamma)}{(\alpha-\beta)(\beta-\gamma)(\alpha-\gamma)} \\ +c \frac{\gamma^{n}(\alpha-\beta)-\beta^{n}(\alpha-\gamma)+\alpha^{n}(\beta-\gamma)}{(\alpha-\beta)(\beta-\gamma)(\alpha-\gamma)}\end{array}\right) f(x-n-1)$

$+c\left(\frac{\gamma^{n+1}(\alpha-\beta)-\beta^{n+1}(\alpha-\gamma)+\alpha^{n+1}(\beta-\gamma)}{(\alpha-\beta)(\beta-\gamma)(\alpha-\gamma)}\right) f(x-n-2)$.

Using (1.2), this gives

$$
\begin{aligned}
f(x)= & T_{n+2} f(x-n)+\left(b T_{n+1}+c T_{n}\right) f(x-n-1) \\
& +c T_{n+1} f(x-n-2),
\end{aligned}
$$

$\forall x \in \mathbb{R}$ and $\forall n \in \mathbb{Z}$.

We use Lemma 3 to prove the following result.

Theorem 4. A function $f: \mathbb{R} \rightarrow X$ is a solution of functional equation (1.3) if and only if there exists $a$ function $h:[-2,1) \rightarrow X$ such that

$$
\begin{aligned}
f(x)= & T\lfloor x\rfloor+2 h(x-\lfloor x\rfloor)+b\left(T_{\lfloor x\rfloor+1}+c T_{\lfloor x\rfloor}\right) h(x-\lfloor x\rfloor-1) \\
& +c T_{\lfloor x\rfloor+1} h(x-\lfloor x\rfloor-2),
\end{aligned}
$$

where $T_{n}$ is given by (1.2).

Proof. If $f(x)$ is a solution of (1.3), then by lemma 3 $f(x)$ satisfies (2.1). Putting $n=\lfloor x\rfloor$ in (2.1), we get

$$
\begin{aligned}
f(x)= & T_{\lfloor x\rfloor+2} f(x-\lfloor x\rfloor)+\left(b T_{\lfloor x\rfloor+1}+c T_{\lfloor x\rfloor}\right) f(x-\lfloor x\rfloor-1) \\
& +c T_{\lfloor x\rfloor+1} f(x-\lfloor x\rfloor-2) .
\end{aligned}
$$

Since $0 \leq x-\lfloor x\rfloor<1, \quad-1 \leq x-\lfloor x\rfloor<0$ and $-2 \leq x-\lfloor x\rfloor-2<-1$, we define a function $h:[-2,1) \rightarrow X$ by $h:=\left.f\right|_{[-2,1)}$, then $f$ is of the form (2.7).

Now we assume that $f$ is a function of the form (2.7) and prove that it is a solution of (1.3).

Consider

$$
\begin{aligned}
& f(x)-a f(x-1)-b f(x-2)-c f(x-3) \\
& =\left(T_{\lfloor x\rfloor+2}-a T_{\lfloor x\rfloor+1}-b T_{\lfloor x\rfloor}-c T_{\lfloor x\rfloor-1}\right) h(x-\lfloor x\rfloor) \\
& +b\left(T_{\lfloor x\rfloor+1}-a T_{\lfloor x\rfloor}-b T_{\lfloor x\rfloor-1}-c T_{\lfloor x\rfloor-2}\right) h(x-\lfloor x\rfloor-1) \\
& +c\left(T_{\lfloor x\rfloor}-a T_{\lfloor x\rfloor-1}-b T_{\lfloor x\rfloor-2}-c T_{\lfloor x\rfloor-3}\right) h(x-\lfloor x\rfloor-1) \\
& +c\left(T_{\lfloor x\rfloor+1}-a T_{\lfloor x\rfloor}-b T_{\lfloor x\rfloor-1}-c T_{\lfloor x\rfloor-2}\right) h(x-\lfloor x\rfloor-2) \\
& =0, \text { for any } x \in \mathbb{R} \text { and arbitrary function } h:[-2,1) \rightarrow X .
\end{aligned}
$$


Therefore (2.7) is a solution of (1.3). Hence the theorem is proved.

We next illustrate this result.

Example 5. Consider the following functional equation

$$
f(x)=\frac{23}{4} f(x-1)-\frac{31}{8} f(x-2)+\frac{5}{8} f(x-3) .
$$

Define the function $h:[-2,1) \rightarrow X$ by

$$
h(x)=x^{3}-\frac{23}{4} x^{2}+\frac{31}{8} x-\frac{5}{8} .
$$

Note tha $\frac{1}{2}, \frac{1}{4}$, and 5 are distinct roots of the characteristic equation corresponding to Tribonacci sequence defined by

$$
\begin{gathered}
T_{n+2}=\frac{23}{4} T_{n+1}-\frac{31}{8} T_{n}+\frac{5}{8} T_{n-1}, \\
T_{0}=0, T_{1}=0, T_{2}=1,
\end{gathered}
$$

$\forall n \in Z$. Therefore (2.7) implies

$$
\begin{aligned}
f(x)= & T\lfloor x\rfloor+2 h(x-\lfloor x\rfloor) \\
& +\left(-\frac{31}{8} T_{\lfloor x\rfloor+1}+\frac{5}{8} T_{\lfloor x\rfloor}\right) h(x-\lfloor x\rfloor-1) \\
& +\frac{5}{8} T_{\lfloor x\rfloor+1} h(x-\lfloor x\rfloor-2),
\end{aligned}
$$

where $h(x)$ is given by (2.9).

\section{Hyers-Ulam Stability}

In this section we prove the Hyers-Ulam stability of functional equation (1.3) by assuming that roots $\alpha, \beta$ and $\gamma$ are distinct and $0<|\alpha|,|\gamma|<1,|\beta|>1$. We first prove the lemma required to prove Hyers-Ulam stability of functional equation (1.3).

Lemma 6. If a function $f: \mathbb{R} \rightarrow X$ satisfies,

$$
\|f(x)-a f(x-1)-b f(x-2)-c f(x-3)\| \leq \epsilon, \forall x \in \mathbb{R},
$$

for some $\epsilon \geq 0$ and $\alpha, \beta$ and $\gamma$ are distinct roots of (2.2) such that $0<|\alpha|, \quad|\gamma|<1, \quad|\beta|>1$, then there exists Tribonacci functions $F_{1}, F_{2}, F_{3}: \mathbb{R} \rightarrow X$ defined by

$$
\begin{aligned}
F_{1}(x)= & \lim _{n \rightarrow \infty} \gamma^{n}[f(x-n) \\
& -(\alpha+\beta) f(x-n-1)+(\alpha \beta) f(x-n-2)], \\
F_{2}(x)= & \lim _{n \rightarrow \infty} \alpha^{n}[f(x-n) \\
& -(\beta+\gamma) f(x-n-1)+(\beta \gamma) f(x-n-2)]
\end{aligned}
$$

and

$$
\begin{aligned}
F_{3}(x)= & \lim _{n \rightarrow \infty} \beta^{-n}[f(x+n) \\
& -(\alpha+\gamma) f(x+n-1)+(\alpha \gamma) f(x+n-2),
\end{aligned}
$$

such that

$$
\begin{array}{r}
\| f(x)-(\alpha+\beta) f(x-1)+(\alpha \beta) f(x-2) \\
-F_{1}(x) \| \leq \frac{\epsilon}{1-|\gamma|}, \\
\| f(x)-(\beta+\gamma) f(x-1)+(\beta \gamma) f(x-2) \\
-F_{2}(x) \| \leq \frac{\epsilon}{1-|\alpha|}
\end{array}
$$

and

$$
\begin{gathered}
\left\|F_{3}(x)-(f(x)-(\alpha+\gamma) f(x-1)+(\alpha \gamma) f(x-2))\right\| \\
\leq \frac{\epsilon}{|\beta|-1},
\end{gathered}
$$

$\forall x \in \mathbb{R}$.

Proof. Using (2.4) with $n=1$ in given condition on $f$, we have

$$
\begin{aligned}
& \| f(x)-(\alpha+\beta) f(x-1)+(\alpha \beta) f(x-2) \\
& -\gamma[f(x-1)-(\alpha+\beta) f(x-2)++(\alpha \beta) f(x-3)] \| \leq \epsilon .
\end{aligned}
$$

Replacing $x$ by $x-k$, we have

$\| f(x-k)-(\alpha+\beta) f(x-k-1)+(\alpha \beta) f(x-k-2)$

$-\gamma[f(x-k-1)-(\alpha+\beta) f(x-k-2)+(\alpha \beta) f(x-k-3)] \| \leq \epsilon$.

Multiplying both side by $|\gamma|^{k}$,

$\| \gamma^{k}[f(x-k)-(\alpha+\beta) f(x-k-1)+(\alpha \beta) f(x-k-2)]$

$-\gamma^{k+1}[f(x-k-1)-(\alpha+\beta) f(x-k-2)+(\alpha \beta) f(x-k-3)] \|$

$\leq|\gamma|^{k} \epsilon, \forall x \in \mathbb{R}$ and $\forall k \in \mathbb{Z}$.

Further,

$$
\begin{gathered}
\| f(x)-(\alpha+\beta) f(x-1)+(\alpha \beta) f(x-2) \\
-\gamma^{n}[f(x-n)-(\alpha+\beta) f(x-n-1)+(\alpha \beta) f(x-n-2)] \| \\
\leq \sum_{k=0}^{n-1} \| \gamma^{k}[f(x-k)-(\alpha+\beta) f(x-k-1)+(\alpha \beta) f(x-k-2)] \\
-\gamma^{k+1}[f(x-k-1)-(\alpha+\beta) f(x-k-2)+(\alpha \beta) f(x-k-3)] \| \\
\leq \sum_{k=0}^{n-1}|\gamma|^{k} \epsilon, \forall x \in \mathbb{R} \text { and } \forall k \in \mathbb{Z} .
\end{gathered}
$$

Therefore

$$
\begin{aligned}
& \| f(x)-(\alpha+\beta) f(x-1)+(\alpha \beta) f(x-2) \\
& -\gamma^{n}[f(x-n)-(\alpha+\beta) f(x-n-1)+(\alpha \beta) f(x-n-2)] \| \\
& \leq \sum_{k=0}^{n-1}|\gamma|^{k} \epsilon, \forall x \in \mathbb{R}, \forall k \in \mathbb{Z} \text { and } \forall n \in \mathbb{N} .
\end{aligned}
$$

Thus for $0<|\gamma|<1$ and for any $x \in \mathbb{R},(3.5)$ implies that the sequence $\left\{\gamma^{n}[f(x-n)-(\alpha+\beta) f(x-n-1)+(\alpha \beta) f(x-n-2)]\right\}$ is a cauchy sequence.

Therefore, since $X$ is Banach space, we can define a function $F_{1}: \mathbb{R} \rightarrow X$ by

$$
\begin{aligned}
F_{1}(x)= & \lim _{n \rightarrow \infty} \gamma^{n}[f(x-n) \\
& -(\alpha+\beta) f(x-n-1)+(\alpha \beta) f(x-n-2)] .
\end{aligned}
$$

We now prove that $F_{1}(x)$ satisfies (1.3). 
Consider,

$$
\begin{aligned}
& a F_{1}(x-1)+b F_{1}(x-2)+c F_{1}(x-3) \\
&= a \gamma^{-1} \lim _{n \rightarrow \infty} \gamma^{n+1}[f(x-(n+1)) \\
&-(\alpha+\beta) f(x-(n+2))+(\alpha \beta) f(x-(n+3))] \\
&+ b \gamma^{-2} \lim _{n \rightarrow \infty} \gamma^{n+2}[f(x-(n+2)) \\
&-(\alpha+\beta) f(x-(n+3))+(\alpha \beta) f(x-(n+4))] \\
&+ c \gamma^{-3} \lim _{n \rightarrow \infty} \gamma^{n+3}[f(x-(n+3)) \\
&-(\alpha+\beta) f(x-(n+4))+(\alpha \beta) f(x-(n+5))] \\
&+ F_{1}(x)\left(a \gamma^{-1}+b \gamma^{-2}+c \gamma^{-3}\right), \\
&= F_{1}(x), \forall x \in \mathbb{R} .
\end{aligned}
$$

Hence $F_{1}$ is a Tribonacci function.

Now taking $n \rightarrow \infty$, (3.5) implies

$$
\begin{aligned}
& \left\|f(x)-(\alpha+\beta)(x-1)+(\alpha \beta) f(x-2)-F_{1}(x)\right\| \\
& \leq \lim _{n \rightarrow \infty} \sum_{k=0}^{n-1}\left|\gamma^{k}\right| \epsilon=\frac{\epsilon}{1-|\gamma|} .
\end{aligned}
$$

Similarly since $0<|\alpha|<1$, using equation (2:6) with $n=1$ we can prove that there exists a Tribonacci function $F_{2}: \mathbb{R} \rightarrow X$ given by

$$
\begin{aligned}
F_{2}(x)= & \lim _{n \rightarrow \infty} \alpha^{n}[f(x-n) \\
& -(\beta+\gamma) f(x-n-1)+(\beta \gamma) f(x-n-2)]
\end{aligned}
$$

such that

$$
\begin{aligned}
& \left\|f(x)-(\beta+\gamma) f(x-1)+(\beta \gamma) f(x-2)-F_{2}(x)\right\| \\
& \leq \lim _{n \rightarrow \infty} \sum_{k=0}^{n-1}\left|\alpha^{k}\right| \epsilon=\frac{\epsilon}{1-|\alpha|} .
\end{aligned}
$$

From equation (2.5) with $n=1$, it follows that

$$
\begin{aligned}
& \| f(x)-(\alpha+\gamma) f(x-1)+(\alpha \gamma) f(x-2) \\
& -\beta[f(x-1)-(\alpha+\gamma) f(x-2)+(\alpha \gamma) f(x-3)] \| \leq \epsilon .
\end{aligned}
$$

Replacing $x$ by $x+k$ and multiplying both sides by $|\beta|^{-k}$, we get

$\| \beta^{-k}[f(x+k)-(\alpha+\gamma) f(x+k-1)+(\alpha \gamma) f(x+k-2)]$ $-\beta^{-k+1}[f(x+k-1)-(\alpha+\gamma) f(x+k-2)+(\alpha \gamma) f(x+k-3)] \|$

$\leq|\beta|^{-k} \epsilon, \forall x \in I R$ and $\forall k \in \mathbb{Z}$.

For $n \in \mathbb{N}$,

$$
\begin{aligned}
& \| \beta^{-n}[f(x+n)-(\alpha+\gamma) f(x+n-1)+(\alpha \gamma) f(x+n-2)] \\
& -[f(x)-(\alpha+\gamma) f(x-1)+(\alpha \gamma) f(x-2)] \| \\
& \leq \sum_{k=1}^{n} \| \beta^{-k}[f(x+k)-(\alpha+\gamma) f(x+k-1)+(\alpha \gamma) f(x+k-2)] \\
& -\beta^{-k-1}[f(x+k-1)-(\alpha+\gamma) f(x+k-2)+(\alpha \gamma) f(x+k-3)] \| \\
& \leq \sum_{k=1}^{n}|\beta|^{-k} \epsilon .
\end{aligned}
$$

Thus, for all $x \in \mathbb{R}$ and $\forall_{n} \in \mathbb{N}$,

$$
\begin{aligned}
& \| \beta^{-n}[f(x+n)-(\alpha+\gamma) f(x+n-1)+(\alpha \gamma) f(x+n-2)] \\
& -[f(x)-(\alpha+\gamma) f(x-1)+(\alpha \gamma) f(x-2)] \| \\
& \quad \leq \sum_{k=1}^{n}|\beta|^{-k} \epsilon .
\end{aligned}
$$

Equation (3.7) implies that

$$
\left\{\beta^{-n}[f(x+n)-(\alpha+\gamma) f(x+n-1)+(\alpha \gamma) f(x+n-2)]\right\}
$$

is a cauchy sequence for any fixed $x \in \mathbb{R}$. Since $X$ is a Banach space, we can define a function $F_{3}: \mathbb{R} \rightarrow X$ by

$$
\begin{aligned}
F_{3}(x)= & \lim _{n \rightarrow \infty} \beta^{-n}[f(x+n) \\
& -(\alpha+\gamma) f(x+n-1)+(\alpha \gamma) f(x+n-2) .
\end{aligned}
$$

Now Consider,

$$
\begin{aligned}
& a F_{3}(x-1)+b F_{3}(x-2)+c F_{3}(x-3) \\
&= a \beta^{-1} \lim _{n \rightarrow \infty} \beta^{-n+1}[f(x+(n-1)) \\
&-(\alpha+\gamma) f(x+(n-2))+(\alpha \gamma) f(x+(n-3))] \\
&+ b \beta^{-2} \lim _{n \rightarrow \infty} \beta^{n+2}[f(x+(n-2)) \\
&-(\alpha+\gamma) f(x+(n-3))+(\alpha \gamma) f(x+(n-4))] \\
&+ c \beta^{-3} \lim _{n \rightarrow \infty} \beta^{n+3}[f(x+(n-3)) \\
&-(\alpha+\gamma) f(x+(n-4))+(\alpha \gamma) f(x+(n-5)) \\
&= F_{3}(x)\left(a \beta^{-1}+b \beta^{-2}+c \beta^{-3}\right) \\
&= F_{3}(x), \forall x \in \mathbb{R} .
\end{aligned}
$$

Therefore $F_{3}(x)$ is a Tribonacci function. So as $n \rightarrow \infty$, we have

$$
\begin{gathered}
\left\|F_{3}(x)-(f(x)-(\alpha+\gamma) f(x-1)+(\alpha \gamma) f(x-2))\right\| \\
\leq \lim _{n \rightarrow \infty} \sum_{k=1}^{n-1}|\beta|^{-k} \epsilon=\frac{\epsilon}{|\beta|-1}, \forall x \in I R .
\end{gathered}
$$

We prove the following theorem using Lemma 6 .

Theorem 7. If a function $f: \mathbb{R} \rightarrow X$ satisfies the inequality,

$$
\|f(x)-a f(x-1)-b f(x-2)-c f(x-3)\| \leq \epsilon, \forall x \in \mathbb{R}(3.8)
$$

and for some $\epsilon \geq 0$, then there exists a unique solution function $F: \mathbb{R} \rightarrow X$ of the functional equation (1.3) such that

$$
\begin{aligned}
& \|f(x)-F(x)\| \\
& \leq \frac{\epsilon}{|\alpha-\beta \| \beta-\gamma||\alpha-\gamma|}\left(\frac{(|\alpha|-|\beta|)|\gamma|^{2}}{1-|\gamma|}+\frac{(|\alpha|-|\gamma|)|\beta|^{2}}{|\beta|-1}\right. \\
& \left.+\frac{(|\beta|-|\gamma|)|\alpha|^{2}}{1-|\alpha|}\right), \forall x \in I R .
\end{aligned}
$$

Proof. From (3.1), (3.2) and (3.3), we see that 


$$
\begin{aligned}
& \left.\| f(x)-\frac{\left[(\alpha-\beta) \gamma^{2} F_{1}(x)-(\alpha-\gamma) \beta^{2} F_{3}(x)+(\beta-\gamma) \alpha^{2} F_{2}(x)\right]}{(\alpha-\beta)(\beta-\gamma)(\alpha-\gamma)}\right] \\
& =\frac{1}{|\alpha-\beta \| \beta-\gamma||\alpha-\gamma|}\left[\|(\alpha-\beta) \gamma^{2}\right. \\
& \left.\left(f(x)-(\alpha+\beta) f(x-1)+(\alpha \beta) f(x-2)-F_{1}(x)\right) \|\right] \\
& +\frac{1}{|\alpha-\beta||\beta-\gamma||\alpha-\gamma|}\left[\left(\mid(\beta-\gamma) \alpha^{2}\right.\right. \\
& \left.\left(f(x)-(\beta+\gamma) f(x-1)+(\beta \gamma) f(x-2)-F_{2}(x)\right) \mid\right] \\
& +\frac{1}{|\alpha-\beta \| \beta-\gamma||\alpha-\gamma|}\left[\mid(\alpha-\gamma) \beta^{2}\right. \\
& \left.\left[F_{3}(x)-(f(x)-(\alpha+\gamma) f(x-1)+(\alpha \gamma) f(x-2))\right] \mid\right] \\
& \leq \frac{\epsilon \quad}{\leq}\left(\frac{(\alpha-\beta)|\gamma|^{2}}{1-|\gamma|}+\frac{(\beta-\gamma)|\alpha|^{2}}{1-|\alpha|}\right. \\
& \left.\quad+\frac{(\alpha-\gamma)|\beta|^{2}}{|\beta|-1}\right), \forall x \in I R .
\end{aligned}
$$

We now define a function $F: \mathbb{R} \rightarrow X$ by

$$
F(x)=\frac{(\alpha-\beta) \gamma^{2} F_{1}(x)-(\alpha-\gamma) \beta^{2} F_{3}(x)+(\beta-\gamma) \alpha^{2} F_{2}(x)}{(\alpha-\beta)(\beta-\gamma)(\alpha-\gamma)},
$$

$\forall x \in I R$.

Consider,

$$
\begin{aligned}
& a F(x-1)-b F(x-2)-c F(x-3) \\
& =a \frac{(\alpha-\beta) \gamma^{2} F_{1}(x-1)-(\alpha-\gamma) \beta^{2} F_{3}(x-1)+(\beta-\gamma) \alpha^{2} F_{2}(x-1)}{(\alpha-\beta)(\beta-\gamma)(\alpha-\gamma)} \\
& +b \frac{(\alpha-\beta) \gamma^{2} F_{1}(x-2)-(\alpha-\gamma) \beta^{2} F_{3}(x-2)+(\beta-\gamma) \alpha^{2} F_{2}(x-2)}{(\alpha-\beta)(\beta-\gamma)(\alpha-\gamma)} \\
& +c \frac{(\alpha-\beta) \gamma^{2} F_{1}(x-3)-(\alpha-\gamma) \beta^{2} F_{3}(x-3)+(\beta-\gamma) \alpha^{2} F_{2}(x-3)}{(\alpha-\beta)(\beta-\gamma)(\alpha-\gamma)} \\
& =\frac{(\alpha-\beta) \gamma^{2} F_{1}(x)-(\alpha-\gamma) \beta^{2} F_{3}(x)+(\beta-\gamma) \alpha^{2} F_{2}(x)}{(\alpha-\beta)(\beta-\gamma)(\alpha-\gamma)} \\
& =F(x), \forall x \in I R .
\end{aligned}
$$

Therefore $F$ is a solution of (1.3). Now we prove the uniqueness of $F(x)$.

Assume that $F, \hat{F}: \mathbb{R} \rightarrow X$ are solutions of (1.3) and that there exist positive constants $\mathrm{C}_{1}$ and $\mathrm{C}_{2}$ such that $\|f(x)-F(x)\| \leq C_{1}$ and $\|f(x)-\widehat{F}(x)\| \leq C_{2}$ for all $x \in \mathbb{R}$.

Therefore by Theorem 4, there exist $h, g:[-2,1) \rightarrow X$ such that

$$
\begin{aligned}
F(x)= & T\lfloor x\rfloor+2 \\
& +\left(c T_{\lfloor x\rfloor+1} h(x-\lfloor x\rfloor)+\left(b T_{\lfloor x\rfloor+1}+c T\lfloor x\rfloor\right) h(x-\lfloor x\rfloor-2)\right.
\end{aligned}
$$

and

$$
\begin{aligned}
\hat{F}(x)= & T\lfloor x\rfloor+2 g(x-\lfloor x\rfloor)+\left(b T_{\lfloor x\rfloor+1}+c T_{\lfloor x\rfloor}\right) g(x-\lfloor x\rfloor-1) \\
& +\left(c T_{\lfloor x\rfloor+1}\right) g(x-\lfloor x\rfloor-2),
\end{aligned}
$$

for any $x \in \mathbb{R}$.

Fix $t$ with $0 \leq t<1$. It then follows from (3.9) and (3.10) that

$$
\begin{aligned}
& \| T_{n+2}(h(t)-g(t))+\left(b T_{n+1}+c T_{n}\right)(h(t-1)-g(t-1)) \\
& +\left(c T_{n+1}\right)(h(t-2)-g(t-2)) \| \\
& =\|F(n)-\widehat{F}(n)\| \\
& \leq\|F(n+t)-f(n+t)\|+\|f(n+t)-\widehat{F}(n+t)\| \\
& \leq C_{1}+C_{2}, \text { for each } n \in \mathbb{Z} .
\end{aligned}
$$

That is,

$$
\begin{aligned}
& \| T_{n+2}(h(t)-g(t))+\left(b T_{n+1}+c T_{n}\right)(h(t-1)-g(t-1)) \\
& +\left(c T_{n+1}\right)(h(t-2)-g(t-2)) \| \\
& \leq C_{1}+C_{2} .
\end{aligned}
$$

This implies

$$
\begin{gathered}
\| \frac{(\alpha-\beta) \gamma^{n+2}-(\alpha-\gamma) \beta^{n+2}+(\beta-\gamma) \alpha^{n+2}}{(\alpha-\beta)(\beta-\gamma)(\alpha-\gamma)}(h(t)-g(t)) \\
+\left(b \frac{(\alpha-\beta) \gamma^{n+1}-(\alpha-\gamma) \beta^{n+1}+(\beta-\gamma) \alpha^{n+1}}{(\alpha-\beta)(\beta-\gamma)(\alpha-\gamma)}\right. \\
\left.+c \frac{(\alpha-\beta) \gamma^{n}-(\alpha-\gamma) \beta^{n}+(\beta-\gamma) \alpha^{n}}{(\alpha-\beta)(\beta-\gamma)(\alpha-\gamma)}\right)(h(t-1)-g(t-1)) \\
+\left(c \frac{(\alpha-\beta) \gamma^{n+1}-(\alpha-\gamma) \beta^{n+1}+(\beta-\gamma) \alpha^{n+1}}{(\alpha-\beta)(\beta-\gamma)(\alpha-\gamma)}\right) \\
(h(t-2)-g(t-2)) \| \\
\leq C_{1}+C_{2} .
\end{gathered}
$$

Dividing both sides by $|\beta|^{n}$ and by letting $n \rightarrow \infty$, we obtain

$$
\begin{aligned}
& \quad \|-(\alpha-\gamma) \beta^{2}(h(t)-g(t)) \\
& \quad+(-b(\alpha-\gamma) \beta-c(\alpha-\gamma))(h(t-1)-g(t-1)) \\
& \quad-c(\alpha-\gamma) \beta(h(t-2)-g(t-2))=0 \\
& \therefore \| \beta^{2}(h(t)-g(t))+(b \beta+c)(h(t-1)-g(t-1)) \\
& +c \beta(h(t-2)-g(t-2)) \|=0 .
\end{aligned}
$$

Analogously, if we divide both sides of by $|\alpha|^{n}$ and $|\gamma|^{n}$ and let $n \rightarrow-\infty$, then we get

$$
\begin{aligned}
& \| \alpha^{2}(h(t)-g(t))+(b \alpha+c)(h(t-1)-g(t-1)) \\
& +c \alpha(h(t-2)-g(t-2)) \|=0
\end{aligned}
$$

and 


$$
\begin{aligned}
& \| \gamma^{2}(h(t)-g(t))+(b \gamma+c)(h(t-1)-g(t-1)) \\
& +c \gamma(h(t-2)-g(t-2)) \|=0 .
\end{aligned}
$$

Rewritting equations (3.12), (3.13) and (3.14) in matrix form, we get

$$
\left[\begin{array}{ccc}
\gamma^{2} & b \gamma+c & c \gamma \\
\alpha^{2} & b \alpha+c & c \alpha \\
\beta^{2} & b \beta+c & c \beta
\end{array}\right]\left[\begin{array}{c}
(h(t)-g(t)) \\
(h(t-1)-g(t-1)) \\
(h(t-2)-g(t-2))
\end{array}\right]=\left[\begin{array}{l}
0 \\
0 \\
0
\end{array}\right] .
$$

Note that since $c \neq 0$ and $\alpha, \beta, \gamma$ are distinct roots,

$$
\left|\begin{array}{lll}
\gamma^{2} & b \gamma+c & c \gamma \\
\alpha^{2} & b \alpha+c & c \alpha \\
\beta^{2} & b \beta+c & c \beta
\end{array}\right|=c^{2}\left|\begin{array}{lll}
\gamma^{2} & 1 & \gamma \\
\alpha^{2} & 1 & \alpha \\
\beta^{2} & 1 & \beta
\end{array}\right|=c^{2}(\alpha-\gamma)(\beta-\gamma)(\alpha-\beta) \neq 0 .
$$

Therefore (3.15) has only trivial solution and we have, $h(t)=g(t), h(t-1)=g(t-1), h(t-2)=g(t-2), \forall t \in[0,1)$.

That is, $h(x)=g(x)$, for all $x \in[-2,1)$. Therefore, we conclude that $F(x)=\hat{F}(x)$ for all $x \in \mathbb{R}$.

We illustrate this result.

\section{Example 8. Consider the functional equation}

$$
f(x)=\frac{23}{4} f(x-1)-\frac{31}{8} f(x-2)+\frac{5}{8} f(x-3)
$$

and Tribonacci recurrence relation associated to it.

$$
\begin{aligned}
& T_{n+2}=\frac{23}{4} T_{n+1}-\frac{31}{8} T_{n}+\frac{5}{8} T_{n-1}, \\
& T_{0}=0, T_{1}=0, T_{2}=1, \forall n \in Z .
\end{aligned}
$$

Roots of the equation $\lambda^{3}-\frac{23}{4} \lambda^{2}+\frac{31}{8} \lambda^{1}+\frac{5}{8}=0$ are $\frac{1}{2}, \frac{1}{4}$ and 5. Let $\alpha=\frac{1}{2}, \beta=5$ and $\gamma=\frac{1}{4}$. Note that roots $\alpha$, $\beta, \gamma$ are distinct and $|\alpha|<1,|\gamma|<1$ and $|\beta|>1$.

Hence the solution is given by

$$
F(x)=\frac{(\alpha-\beta) \gamma^{2} F_{1}(x)-(\alpha-\gamma) \beta^{2} F_{3}(x)+(\beta-\gamma) \gamma^{2} F_{2}(x)}{(\alpha-\beta)(\beta-\gamma)(\alpha-\gamma)}
$$

where

$$
\begin{aligned}
F_{1}(x)= & \lim _{n \rightarrow \infty}\left(\frac{1}{4}\right)^{n}[f(x-n) \\
& -(\alpha+\beta) f(x-n-1)+(\alpha \beta)(x-n-2)] \\
F_{2}(x)= & \lim _{n \rightarrow \infty}\left(\frac{1}{2}\right)^{n}[f(x-n) \\
& -(\beta+\gamma) f(x-n-1)+(\beta \gamma)(x-n-2) \\
F_{2}(x)= & \lim _{n \rightarrow \infty}(5)^{-n}[f(x+n) \\
& -(\alpha+\gamma) f(x+n-1)+(\alpha \gamma)(x+n-2) .
\end{aligned}
$$

\section{Therefore}

$$
\begin{aligned}
& F(x)=\frac{\left(\frac{-9}{2} \times \frac{1}{16}\right) F_{1}(x)-\left(\frac{1}{4} \times 25\right) F_{3}(x)+\left(\frac{19}{4} \times \frac{1}{4}\right) F_{2}(x)}{\left(\frac{-9}{2}\right)\left(\frac{19}{4}\right)\left(\frac{1}{4}\right)} \\
& =\frac{9 F_{1}(x)+200 F_{3}(x)-38 F_{2}(x)}{171}
\end{aligned}
$$

and $\|f(x)-F(x)\| \leq \frac{114}{171} \epsilon$.

\section{Conclusion}

Hyers-Ulam stability of generalized Tribonacci functional equation is discussed after obtaining the solution of generalized Tribonacci functional equation in terms of generalized Tribonacci numbers.

\section{References}

[1] Hyers D.. On the stability of the linear functional Equation, Proc. Natl. Acad. Sci. USA, 27(1941). 222-224.

[2] Gordji M., Naderi M., Rassias Th.. Solution and Stability of Tribonacci functional equation in Non-Archmedean Banach spaces. ScienceDirect, Chaos and Fractrals 39(2009). 1005-1019.

[3] Naderi M., Gordji M.. Hyers-Ulam Stability of Fibonacci functional equation in Modular Functional Spaces. Journal of Mathematics and Computer Science, 10(2014). 1-6.

[4] Gordji M., Divandari A., Rostamian M., Park C. and Shin D. Hyers-Ulam Stability of Tribonacci functional equation in 2-normed spaces. Journal of Computational Analysis and Applications, 16 (2014), 503-508

[5] Rassias M.. On the stability of functional equations and a problem of Ulam, Acta Applicandae Mathematicae, 62(1) (2000). 23-130.

[6] Jung S. M.. Hyers-Ulam-Rassias Stability of Functional Equations in Mathematical Analysis, Hadronic Press, Inc., Florida. 2001.

[7] Jung S. M.. Hyers-Ulam-Stability of Fibonacci Functional Equation, Bulletin of Iranian Mathematica Society, 35(2) (2009). 217-227.

[8] Jung S. M.. Functional Equation $f(x)=p f(x-1)-q f(x-2)$ and its Hyers-Ulam Stability, Hindawi Publishing Corporation Journal of Inequalities and Applications Volume 2009, Article ID 181678.

[9] Jung S. M. and Rassias M.. A linear Functional Equation of Third order Associated with the Fibonacci Numbers, Hindawi Publishing Corporation Abstract and Applied Analysis Volume 2014, Article ID 137468.

[10] Bidkham M. and Hosseini M.. Hyers-Ulam Stability of $k$ Fibonacci Functional Equation, Int. J. Nonlinear Anal. Appl.2 (1) (2011) 42-49.

[11] Stevic S. Bounded solutions of a class of difference equations in Banach spaces producing controlled chaos, Chaos, Solitonsand fractals, 35(2) (2008). 238-245.

[12] Kuhapatanakul K. The Generalized Tribonacci p-numbers and Applications, East- West J. of Mathematics: 14(2) (2012). 144-153.

[13] Lather R., Ashish, Kumar M.. On the stability of Tribonacci and $k$-Tribonacci functional equations in Modular space, International Journal of Pure and Applied Mathematics, 104(2) (2015), 265-284.

[14] W. Hayes and K.R. Jackson. A survey of shadowing methods for numerical solutions of ordinary differential equations, Applied Numerical Mathematics 53(2-4)(2005), 299-321.

[15] D. Bozkurt, C. M. da Fonseca and F.Yilmaz, The determinant of a circulant and skew-circulant matrices with Tribonacci numbers., Mathematical Sciences and applications e-notes, 2(2) (2014), 67-75. 